\section{THU0430 PHARMACOKINETICS, PHARMACODYNAMICS, AND TOLERABILITY OF CONCOMITANT MULTIPLE DOSE ADMINISTRATION OF VERINURAD (RDEA3170) AND FEBUXOSTAT IN HEALTHY ADULT MALE SUBJECTS}

J.T. VanderLugt ${ }^{1}$, M. Gillen ${ }^{2}$, X. Yang ${ }^{3}$, J. Hall ${ }^{3} .{ }^{1}$ Jasper Clinical Research \& Development, Kalamazoo, MI; ${ }^{2}$ AstraZencea, Gaithersburg, MD; ${ }^{3}$ Ardea Biosciences, Inc., San Diego, CA, United States

Background: Verinurad (RDEA3170) is a novel selective uric acid reabsorption inhibitor in clinical development for the treatment of gout and asymptomatic hyperuricemia.

Objectives: This Phase 1, single-blind, multiple dose, drug-drug interaction (DDI) study evaluated the pharmacokinetics (PK), pharmacodynamics, and tolerability of verinurad in combination with febuxostat (FBX) in healthy male volunteers. Methods: Subjects were randomized to receive once-daily doses of FBX or verinurad or placebo alone for 7 days, FBX + verinurad or FBX + placebo on days $8-14$, and the alternative single agent (FBX or verinurad or placebo) on days 15-21. Subjects received either the combination of verinurad $10 \mathrm{mg}+\mathrm{FBX} 40$ $\mathrm{mg}$ or verinurad $2.5 \mathrm{mg}+\mathrm{FBX} 80 \mathrm{mg}$. Serial plasma/serum and urine samples were drawn at predetermined time points on Days 7, 14 and 21, and assayed for verinurad, FBX, and uric acid. Baseline samples were drawn on Day -1 . Safety was assessed by adverse event (AE) reports, laboratory tests, vital signs, and electrocardiograms (ECGs).

Results: Of 23 randomized subjects, 20 completed the study. FBX $40 \mathrm{mg}$ had no apparent effect on the plasma $C_{\max }$ and AUC for verinurad $10 \mathrm{mg}$, whereas FBX $80 \mathrm{mg}$ increased the plasma $\mathrm{C}_{\max }$ and AUC for verinurad $2.5 \mathrm{mg}$ by $25 \%$ and $33 \%$, respectively. Verinurad had no effect on FBX PK. Renal clearance of verinurad was unchanged by FBX.

The mean maximal reduction in serum uric acid (sUA) was $76 \%$ with verinurad $10 \mathrm{mg}+$ FBX $40 \mathrm{mg}$ compared with verinurad $10 \mathrm{mg}$ (56\%) or FBX $40 \mathrm{mg}(49 \%)$ alone (Figure $1 \mathrm{~A}$ ) and was $67 \%$ with verinurad $2.5 \mathrm{mg}+\mathrm{FBX} 80 \mathrm{mg}$ compared with verinurad $2.5 \mathrm{mg}(38 \%)$ or FBX $80 \mathrm{mg}(57 \%)$ alone (Figure 1B). Consistent with the mechanism of action (MOA) of verinurad, 24-hr fractional excretion of uric acid (FEUA) increased (2.5 mg: $7.6 \% ; 10 \mathrm{mg}: 12.8 \%$ ) vs baseline $(6.5 \%$ and $6.0 \%$, respectively). Renal clearance of uric acid (CLuR) increased similarly (2.5 mg: $9.0 \mathrm{~mL} / \mathrm{min} ; 10 \mathrm{mg}: 12.3 \mathrm{~mL} / \mathrm{min}$ ) vs baseline ( 8.3 and $7.3 \mathrm{~mL} / \mathrm{min}$, respectively). The increases were maintained for 24 hours with verinurad $10 \mathrm{mg}+\mathrm{FBX} 40 \mathrm{mg}$ (FEUA: 11.8\%; CLuR: 13.6mL/min). Consistent with its MOA, FBX $40 \mathrm{mg}$ and $80 \mathrm{mg}$ decreased the amount of uric acid excreted in urine $(246$ and $221 \mathrm{mg}$, respectively, vs baseline: 695 and $818 \mathrm{mg}$ ), FEUA (4.79 and $4.18 \%$, respectively, vs baseline: 6.31 and $6.54 \%$ ), and CLuR $(4.91$ and $4.96 \mathrm{~mL} / \mathrm{min}$, respectively, vs baseline: 7.71 and $8.73 \mathrm{~mL} / \mathrm{min}$ ). No serious $\mathrm{AEs}$, discontinuations due to $\mathrm{AEs}$, or clinically significant laboratory or ECG abnormalities were noted during the study.

Figure 1: Mean (SE) percent change from baseline in sUA at steady state following verinurad $10 \mathrm{mg}$ or FBX $40 \mathrm{mg}$ or the combination (A) or verinurad $2.5 \mathrm{mg}$ or FBX $80 \mathrm{mg}$ or the combination (B)
A.

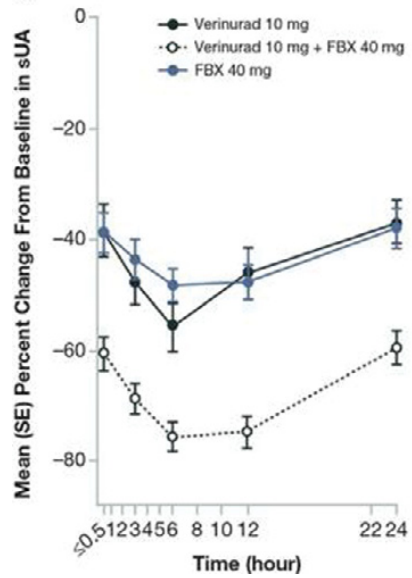

B.

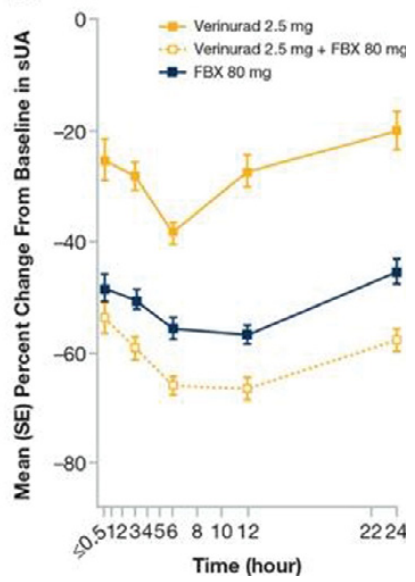

Conclusions: No DDI was found with the verinurad $10 \mathrm{mg}+\mathrm{FBX} 40 \mathrm{mg}$ combination and only a modest one with verinurad $2.5 \mathrm{mg}+\mathrm{FBX} 80 \mathrm{mg}$. Both combinations were safe and well tolerated and resulted in greater reduction of sUA than either verinurad or FBX alone. These results support the continued development of this novel approach for the treatment of gout and hyperuricemia. Disclosure of Interest: J. VanderLugt: None declared, M. Gillen Employee of: AstraZeneca, X. Yang Employee of: Ardea Biosciences, Inc., J. Hall Employee of: Ardea Biosciences, Inc.

DOI: 10.1136/annrheumdis-2017-eular.5264

\section{THU0431 COMPARATIVE EFFECTIVENESS OF ALLOPURINOL VERSUS FEBUXOSTAT FOR PREVENTING INCIDENT RENAL DISEASE IN OLDER ADULTS: AN ANALYSIS OF MEDICARE CLAIMS DATA}

J. Singh, J. Cleveland. University of Alabama at Birmingham, Birmingham, United States

Background: Large scale randomized studies are underway to assess whether compared to placebo, each XO-inhibitor, allopurinol or febuxostat, can prevent renal function loss. This evidence is needed and will confirm their nephroprotective potential. However, neither study will answer a key question: Does the renal protective effect of allopurinol differ from that of febuxostat?

Objectives: To assess the comparative effectiveness of allopurinol vs. febuxostat for preventing incident renal disease in elderly.

Methods: In a retrospective cohort study using Medicare claims data, we included patients newly treated with allopurinol or febuxostat (baseline period of 183 days without either medication). We used 1:5 propensity-matched Cox regression analyses to compare the hazard ratio $(\mathrm{HR})$ of incident renal disease with allopurinol use and allopurinol dose vs. febuxostat (reference category). Sensitivity analyses included multivariable-adjusted regression models.

Results: There were 31,465 new allopurinol or febuxostat treatment episodes in 26,443 patients; 8,570 ended in incident renal disease. Crude rates of incident renal disease per 100,000 person-days were 53 with allopurinol vs. 93 with febuxostat. Crude rates of incident renal disease per 100,000 person-days were lower with higher daily dose: allopurinol $<200,200-299$ and $\geq 300 \mathrm{mg} /$ day with 65, 48 and 43; and febuxostat $40 \mathrm{mg}$ and $80 \mathrm{mg} /$ day with 93 and 89 , respectively. In propensity-matched analyses, compared to febuxostat use, allopurinol use was associated with lower HR of incident renal disease, 0.61 (95\% confidence interval (Cl): $0.49,0.77)$. Compared to febuxostat $40 \mathrm{mg} /$ day, allopurinol doses $<200$, 200-299 and $\geq 300 \mathrm{mg} /$ day were associated with lower HR of incident renal disease, 0.75 (95\% Cl: $0.65,0.86), 0.61(95 \% \mathrm{Cl}: 0.52,0.73)$ and $0.48(95 \%$ $\mathrm{Cl}: 0.41,0.55)$, respectively. Sensitivity analyses using multivariable-adjusted regression confirmed these findings.

Conclusions: Allopurinol was more effective than febuxostat in preventing incident renal disease in elderly patients. Future studies need to examine the mechanism of this renal benefit of allopurinol.

Disclosure of Interest: J. Singh Grant/research support from: Savient, Takeda, Consultant for: Savient, Takeda, Regeneron, Merz, Iroko, Bioiberica, Crealta and Allergan pharmaceuticals, WebMD, UBM LLC and the American College of Rheumatology, J. Cleveland: None declared

DOI: 10.1136/annrheumdis-2017-eular.6837

\section{THU0432 PHARMACOKINETICS, PHARMACODYNAMICS, AND TOLERABILITY OF CONCOMITANT MULTIPLE DOSE ADMINISTRATION OF VERINURAD (RDEA3170) AND ALLOPURINOL IN ADULT MALE SUBJECTS WITH GOUT}

J. Hall ${ }^{1}$, M. Gillen ${ }^{2}$, X. Yang ${ }^{1}$, S. Liu ${ }^{1}$, S. Walker ${ }^{2}$, V. Clauson ${ }^{1}$, M. Kankam ${ }^{3}$ ${ }^{1}$ Ardea Biosciences, Inc., San Diego, $C A ;{ }^{2}$ AstraZeneca, Gaithersburg, MD; ${ }^{3}$ Vince and Associates Clinical Research, Inc., Overland Park, KS, United States

Background: Verinurad (RDEA3170) is a novel selective uric acid reabsorption inhibitor in clinical development for the treatment of hyperuricemia and gout. Objectives: This Phase 1, single-blind, multiple dose, drug-drug interaction study evaluated the pharmacokinetics (PK), pharmacodynamics, and tolerability of verinurad in combination with allopurinol (ALLO) in adult male subjects with gout. Methods: Adult males with gout, aged 18-75 years, with serum uric acid (sUA) $\geq 8 \mathrm{mg} / \mathrm{dL}$ were randomized to receive once-daily oral doses of ALLO $300 \mathrm{mg}$ or verinurad $10 \mathrm{mg}$ alone for 7 days, ALLO $300 \mathrm{mg}$ + verinurad $10 \mathrm{mg}$ on Days $8-14$, and the alternative single agent (verinurad $10 \mathrm{mg}$ or ALLO $300 \mathrm{mg}$ ) on Days 15-21. Colchicine $0.6 \mathrm{mg}$ was taken once daily from day -14 . Serial plasma/serum and urine samples were drawn at predetermined time points on Days 7, 14 and 21 and assayed for verinurad, ALLO, oxypurinol (OXY), colchicine, and uric acid. Baseline samples were drawn on Day -1 . Safety was assessed by adverse event $(\mathrm{AE})$ reports, laboratory tests, vital signs, and electrocardiograms (ECGs)

Results: Subjects $(\mathrm{N}=12)$ were mostly white (58.3\%) with mean (SD) age of 51 (10) years. Following multiple doses, ALLO had no effect on $\mathrm{C}_{\max }$ and AUC of verinurad. ALLO $\mathrm{C}_{\max }$ was increased $33 \%$ but AUC was unaltered by verinurad. The $\mathrm{C}_{\max }$ and AUC for OXY, the active metabolite of ALLO, were reduced $32 \%$ and $38 \%$, respectively, by verinurad. Colchicine plasma exposures were unaltered by verinurad. ALLO had no effect on urinary excretion of verinurad, whereas urinary excretion of OXY was increased $19 \%$ by verinurad.

The mean maximal decrease in SUA was $65 \%$ with verinurad + ALLO compared with verinurad $(51 \%)$ or ALLO (43\%) alone (Figure). Consistent with the mechanism of action (MOA) of verinurad, 24-h fractional excretion of uric acid (FEUA) and clearance of uric acid ( $\mathrm{CL}_{u \mathrm{R}}$ ) were increased in the absence $(9.2 \%$ and $11.5 \mathrm{~mL} / \mathrm{min}$, respectively) or presence of ALLO $(7.9 \%$ and $11.8 \mathrm{~mL} / \mathrm{min})$ vs baseline $(4.5 \%$ and $5.7 \mathrm{~mL} / \mathrm{min})$ or ALLO alone $(3.7 \%$ and $5.0 \mathrm{~mL} / \mathrm{min})$. Consistent with its MOA, ALLO decreased the amount of uric acid excreted in 24-h urine (363 mg) compared with baseline $(683 \mathrm{mg})$, verinurad alone (739 $\mathrm{mg}$ ) or verinurad + ALLO (522 mg) but had no effect on FEUA or CLUR. No 
serious AEs, discontinuations due to AEs, or clinically significant laboratory or ECG abnormalities were noted.

Figure: Mean (SE) percent change from baseline in SUA at steady state following verinurad $10 \mathrm{mg}$ or ALLO $300 \mathrm{mg}$ or in combination

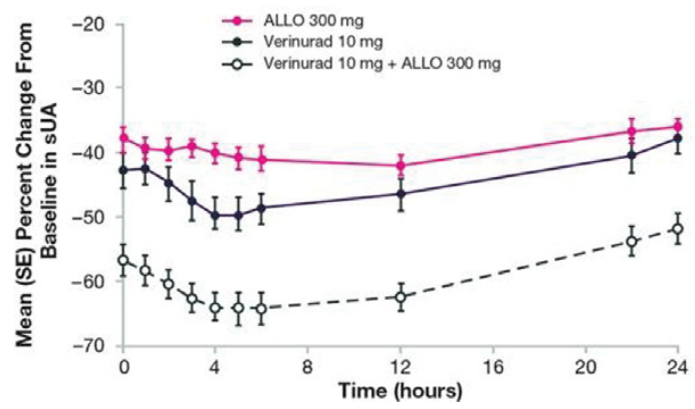

Conclusions: Although a modest drug-drug interaction was found between verinurad and ALLO, the combination was safe and well tolerated at the studied doses and resulted in greater reduction of sUA than either alone. These results support the evaluation of verinurad + ALLO as an alternative once-daily treatment option for hyperuricemia and gout.

Disclosure of Interest: J. Hall Employee of: Ardea Biosciences, Inc., M. Gillen Employee of: AstraZeneca, X. Yang Employee of: Ardea Biosciences, Inc., S. Liu Employee of: Ardea Biosciences, Inc., S. Walker Employee of: AstraZeneca, V. Clauson Employee of: Ardea Biosciences, Inc., M. Kankam: None declared DOI: 10.1136/annrheumdis-2017-eular.5308

\section{THU0433 PHARMACODYNAMIC AND PHARMACOKINETIC STUDY OF VERINURAD (RDEA3170) IN ADULT MALE SUBJECTS WITH MILD, MODERATE, AND SEVERE RENAL IMPAIRMENT: A PHASE 1, OPEN-LABEL STUDY}

W.B. Smith ${ }^{1}$, J. Hall ${ }^{2}$, J.K. Berg ${ }^{3}$, M. Kazimir ${ }^{3}$, A. Yamamoto ${ }^{2}$, S. Walker ${ }^{4}$, C. Lee ${ }^{2}$, T.C. Marbury ${ }^{5} .{ }^{1}$ Volunteer Research Group, Knoxville, TN; ${ }^{2}$ Ardea Biosciences, Inc., San Diego, CA; ${ }^{3}$ DaVita Clinical Research, Minneapolis, MN; ${ }^{4}$ Astrazeneca, Gaithersburg, MD; ${ }^{5}$ Orlando Clinical Research Center, Orlando, FL, United States

Background: Verinurad (RDEA3170) is a high-affinity, selective URAT1 inhibitor in development for the treatment of gout and asymptomatic hyperuricemia.

Objectives: This Phase 1, single-dose, open-label study investigated the pharmacodynamics (PD), pharmacokinetics (PK), and safety of oral verinurad in adult subjects with mild, moderate, or severe renal impairment and matched controls with normal renal function (NCT02219516).

Methods: Adult males aged 18-85 years were enrolled with a screening serum uric acid (sUA) 4.5-10 mg/dL and creatinine clearance calculated by CockcroftGault formula of 60 to $<90 \mathrm{~mL} / \mathrm{min}$ (mild renal impairment), 30 to $<60 \mathrm{~mL} / \mathrm{min}$ (moderate impairment), 15 to $<30 \mathrm{~mL} / \mathrm{min}$ (severe impairment), or $\geq 90 \mathrm{~mL} / \mathrm{min}$ (matched controls). Oral verinurad $15 \mathrm{mg}$ was administered once under fasted conditions. Serial blood and urine samples were taken $30 \mathrm{~min}$ before and up to 72 hours postdose. Safety assessments included laboratory, ECG, and vital sign parameters as well as adverse event (AEs) reporting.

Results: PD data were based on 7-8 subjects per group. Verinurad decreased sUA in all groups, with greatest changes in the normal function and mild renal impairment groups. Mean (SD) maximal \% change in sUA from baseline (Emax) was $-38.3(14.8) \%,-36.9(13.6) \%,-20.5(6.64) \%$, and $-12.6(6.94) \%$, respectively, in the normal function and mild, moderate, and severe renal impairment groups. Increase in the amount of excretion of uric acid due to verinurad treatment decreased in subjects with moderate and severe renal impairment. Plasma Cmax and AUC of verinurad increased with decreasing renal function. Verinurad at the $15 \mathrm{mg}$ dose was well tolerated, with no serious AEs, no subject withdrawals due to AEs, and no Renal Events Adjudication Committee (REAC)-adjudicated renal events during treatment. One patient in each renal impairment group had treatment-emergent AEs considered possibly related to verinurad, which were categorized as gastrointestinal in nature. There were no clinically meaningful changes noted in laboratory values or vital signs.

Conclusions: The sUA lowering effect of verinurad was observed across the spectrum of renal function. Consistent with the verinurad renal-dependent mechanism of action, decreasing sUA lowering was demonstrated with increasing renal impairment. Verinurad safety and tolerability were similar across all stages of renal impairment.

Disclosure of Interest: W. Smith Employee of: Volunteer Research Goup, J. Hall Employee of: Ardea Biosciences, Inc., J. Berg Employee of: DaVita Clinical Research, M. Kazimir Employee of: DaVita Clinical Research, A. Yamamoto Employee of: Ardea Biosciences, Inc., S. Walker Employee of: AstraZeneca, C. Lee Employee of: Ardea Biosciences, Inc., T. Marbury Employee of: Orlando Clinical Research Center

DOI: 10.1136/annrheumdis-2017-eular.5327

\section{THU0434 PHARMACODYNAMIC EFFECTS AND SAFETY OF VERINURAD (RDEA3170) IN COMBINATION WITH FEBUXOSTAT VERSUS FEBUXOSTAT ALONE IN ADULTS WITH GOUT: A PHASE 2A, OPEN-LABEL STUDY}

R. Fleischmann ${ }^{1}$, P. Winkle ${ }^{2}$, J. Hall $^{3}$, S. Valdez ${ }^{3}$, S. Liu ${ }^{3}$, X. Yan ${ }^{3}$, L. Hicks ${ }^{3}$, M. Hernandez-Illas ${ }^{4}$. ${ }^{1}$ University of Texas, Southwestern Medical Center, Metroplex Clinical Research Center, Dallas, TX; ${ }^{2}$ Anaheim Clinical Trials, Anaheim, CA; ${ }^{3}$ Ardea Biosciences, Inc., San Diego, CA; ${ }^{4}$ 4QPS MRA (Miami Clinical Research), Miami, FL, United States

Background: Verinurad (RDEA3170) is a high-affinity, selective URAT1 inhibitor in development for the treatment of gout and asymptomatic hyperuricemia. Objectives: This Phase $2 \mathrm{a}$, randomized, open-label, multicenter study investigated the multiple-dose pharmacodynamics (PD), pharmacokinetics (PK), and safety of oral verinurad in combination with febuxostat versus febuxostat alone in adults with gout (NCT02246673)

Methods: Patients aged $\geq 18$ and $\leq 75$ years with gout and serum uric acid (sUA) $>8 \mathrm{mg} / \mathrm{dL}$ were randomized to 1 of 5 cohorts to receive febuxostat (40 mg and $80 \mathrm{mg}$ ) alone and in combination with verinurad (dose range $2.5 \mathrm{mg}$ to $20 \mathrm{mg} ; 4$ treatment periods per cohort, each treatment period 7 days). Medications were administered once daily $\sim 30 \mathrm{~min}$ after breakfast. Colchicine $0.6 \mathrm{mg}$ for gout flare prophylaxis was initiated at approximately Day -14 (start of urate-lowering therapy [ULT]) washout) or Day -7 if not on ULT. Serial blood and urine samples were measured at preset intervals on Days -1, 1, 7, 14, 21, and 28 for PD and PK endpoints. Safety assessments included adverse events (AEs) and laboratory, electrocardiograms, and vital sign parameters.

Results: Sixty-four patients were randomized ( $n=12-14$ per cohort). Serum PD data pooled across cohorts demonstrated maximal \% decrease in sUA from baseline (Emax) at 8-12 $\mathrm{h}$ after dosing. Addition of verinurad to febuxostat decreased SUA in dose-dependent manner (Figure 1). Greater sUA reductions were observed for dose combinations of verinurad $\geq 5 \mathrm{mg}$ with febuxostat $40 \mathrm{mg}$ versus febuxostat $80 \mathrm{mg}$ alone. The rate of urinary uric acid excretion was reduced by febuxostat alone, but comparable to baseline levels with verinurad combined with febuxostat. Verinurad plasma exposures increased with verinurad dose and were comparable for febuxostat $40 \mathrm{mg}$ and $80 \mathrm{mg}$ doses. No drug-drug interaction on verinurad and febuxostat plasma PK parameters was observed. Verinurad at doses from $2.5 \mathrm{mg}$ to $20 \mathrm{mg}$ was well tolerated, with no serious AEs, withdrawals due to $A E s$, or renal-related events. The most frequent treatment-emergent $A E$ possibly related to study medication was pain in extremity, in 2 patients receiving verinurad. Laboratory values and vital signs showed no clinically meaningful changes. There were no cases of serum creatinine elevation $\geq 1.5 x$ baseline.

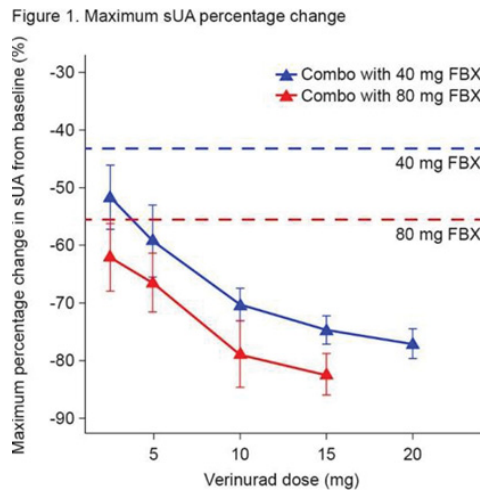

Conclusions: Verinurad coadministered with febuxostat dose-dependently decreased SUA while maintaining urine uric acid levels comparable to baseline. All dose combinations of verinurad and febuxostat in this study were generally well tolerated with no serious AEs or renal-related events during combination treatment.

Disclosure of Interest: R. Fleischmann Grant/research support from: Ardea Biosiences, Inc, P. Winkle Employee of: Anaheim Clinical Trials, J. Hall Employee of: Ardea Biosiences, Inc., S. Valdez Employee of: Ardea Biosiences, Inc., S. Liu Employee of: Ardea Biosiences, Inc., X. Yan Employee of: Ardea Biosiences, Inc., L. Hicks Employee of: Ardea Biosiences, Inc., M. Hernandez-Illas: None declared DOI: 10.1136/annrheumdis-2017-eular.5367

\section{THU0435 PHARMACODYNAMICS, PHARMACOKINETICS, AND SAFETY OF VERINURAD (RDEA3170) IN COMBINATION WITH FEBUXOSTAT VERSUS FEBUXOSTAT ALONE AND VERINURAD ALONE IN JAPANESE ADULTS WITH GOUT OR ASYMPTOMATIC HYPERURICEMIA: A PHASE 2A, OPEN-LABEL STUDY}

M. Shiramoto ${ }^{1}, \mathrm{~S}$. Liu ${ }^{2}$, Z. Shen ${ }^{2}$, J. Hall ${ }^{2} .{ }^{1}$ SOUSEIKAI PS Clinic, Fukuoka, Japan; ${ }^{2}$ Ardea Biosciences, Inc., San Diego, CA, United States

Background: Verinurad (RDEA3170) is a high-affinity URAT1 inhibitor in development for the treatment of gout and asymptomatic hyperuricemia. 